\title{
Advances in stroke treatment are within reach
}

\author{
A Taylor, D le Feuvre, V Mngomezulu, D Royston, R Harrichandparsard, C de Vries, A Winter, F Potgieter
}

Allan Taylor and David le Feuvre are neurosurgeons working in the Division of Neurosurgery, Groote Schuur Hospital and Faculty of Health Sciences, University of Cape Town, South Africa. Victor Mngomezulu is a radiologist and Head of the Department of Radiology in the Faculty of Health Sciences, University of the Witwatersrand, Johannesburg, South Africa. Duncan Royston is a radiologist and partner at Lake, Smit Partners in Durban, South Africa. Rohen Harrichandparsard is a neurosurgeon working at Inkosi Albert Luthuli Hospital and the Division of Neurosurgery, School of Clinical Medicine, College of Health Sciences, Nelson R Mandela School of Medicine, University of KwaZulu-Natal, Durban, South Africa. Coert de Vries is a radiologist and head of the Department of Radiology in the Faculty of Health Sciences, University of the Free State, Bloemfontein, South Africa. Arthur Winter and Francois Potgieter are radiologists and partners at Burger Radiologists in Pretoria, South Africa.

Corresponding author: A Taylor (allan.taylor@uct.ac.za)

Five recent trials have shown that mechanical removal of clot from cerebral arteries after a stroke can achieve a functional independent outcome in up to $60 \%$ of patients. This was an absolute benefit of between $13.5 \%$ and $31 \%$ for patients who had clot removal initiated within 6 hours of symptoms over those who had best medical treatment. Coupled with this, there is a strong drive to develop stroke units internationally and in South Africa. As a starting point, more primary stroke care centres that can administer intravenous thrombolysis are needed. Comprehensive stroke centres that can offer mechanical thrombectomy are available, but more will be required as referral of patients increases. Collaboration of all role-players will ensure that we can deliver training and care at the best level for stroke patients.

S Afr Med J 2016;106(5):454-455. DOI:10.7196/SAMJ.2016.v106i5.10355

Stroke is no longer regarded as an untreatable condition with inevitable long-term morbidity. The current drive to establish more stroke units in South African (SA) public and private hospitals is to be commended. ${ }^{[1]}$ There is robust evidence in the medical literature to indicate that treating stroke patients in a multidisciplinary stroke unit significantly reduces death and dependency. ${ }^{[2]}$ The components of care that are probably responsible for this improved outcome include good nursing care, protocol-driven care with attention to swallowing, hydration, nutrition, early detection of complications of stroke and early rehabilitation.

Intravenous thrombolysis with tissue plasminogen activator (tPA) has become the standard of care in specialised stroke units for selected patients who can be treated within 4.5 hours of onset of symptoms. Irrespective of age or stroke severity, and despite an increased risk of fatal intracranial haemorrhage during the first few days after treatment, intravenous tPA significantly improves the overall odds of a good stroke outcome when delivered within 4.5 hours of stroke onset, with earlier treatment associated with bigger proportional benefits..$^{[3]}$ This treatment should only be provided in stroke centres, where the staff are trained to administer thrombolysis for stroke using a clear protocol with access to early brain imaging, laboratory facilities and neurosurgical advice.

\section{Emerging treatment - mechanical thrombectomy}

Until recently, intravenous tPA has been the only reperfusion therapy proven to reduce disability after acute ischaemic stroke. Although intravenous tPA is effective at recanalising more distal thrombi, it is less successful in dissolving larger more proximal thrombi. ${ }^{[4]}$ Randomised trials published in the past year have shown significant benefit of mechanical thrombectomy within 6 hours of onset over best medical treatment in patients with acute ischaemic stroke due to proximal large-vessel occlusion (terminal internal carotid, middle cerebral and anterior cerebral arteries), with an absolute benefit (modified Rankin score $0-2$ at 90 days) for functional outcome ranging from $13.5 \%$ to $31 \%$ for thrombectomy over best medical treatment. ${ }^{[5-9]}$ The development of more effective devices has allowed reopening of vessels in up to $84.5 \%$ of patients. ${ }^{[10]}$ Currently the most effective option is the use of retrievable stents, which are deployed into the thrombus and then pulled out together with the clot that is occluding the affected vessel. The results of these recently published trials for acute ischaemic stroke herald the beginning of a new era in the treatment of patients with large-vessel occlusion amenable to endovascular intervention.

Mechanical thrombectomy is not without risk. Vessels can be damaged, resulting in rupture and intracranial bleeding, and clot can be fragmented and occlude more distal vessels. Fortunately these complications are infrequent when the procedure is performed by trained interventionists. ${ }^{\left[{ }^{5]}\right.}$ Guidelines and consensus documents in both Europe and the USA recommend this form of treatment in the context of comprehensive stroke centres, where patients with largevessel occlusion of the anterior circulation presenting within 6 hours after symptom onset can be considered for mechanical thrombectomy. For those arriving within 4.5 hours, intravenous thrombolysis is also recommended prior to thrombectomy, unless contraindicated. ${ }^{[1,12]}$ Careful selection of patients by stroke neurologists/physicians in the setting of a stroke centre, optimising time to reperfusion by appropriately trained interventionists, and performance of the procedure in a 'high-volume centre' with regular audit will be critical to providing benefit to patients.

\section{Patient selection}

Re-establishing cerebral perfusion is only of benefit if brain tissue is not completely infarcted. Identifying patients who have ischaemic but still viable tissue, the penumbra, is important, as is not selecting patients with large established infarcts. Patients with large infarcts have an increased risk of reperfusion bleeding. Various computed tomography and magnetic resonance imaging techniques are currently used in conjunction with clinical findings when selecting patients. ${ }^{[6,9]}$ This triage process requires expertise in image 
interpretation and clinical evaluation and must be done in organised stroke centres with stroke physicians.

\section{Neurointerventional training}

Unlike specialties and subspecialties registered with the Health Professions Council of South Africa and examined via the Colleges of Medicine, the training requirements for new disciplines such as neurointervention are not yet well defined. Training standards aligned with international norms have been adopted by the academic teaching units in this country and form the basis for peer recognition for proficiency as a neurointerventionist. ${ }^{[13]}$ Such candidates are expected to undergo practical training at an established unit for 1 - 2 years, depending on prior clinical experience and exposure to diagnostic neuroradiology and clinical neurosciences. Training should include a logbook with a minimum number of supervised procedures. The South African Neurointervention Society expects its members to participate in annual peer review meetings, and adherence to evidence-based practice is strongly encouraged. Assuming that any doctor working in the endovascular field is able to do an intracranial intervention effectively and safely without the recommended training is flawed. Participation in short courses, workshops and industrydriven programmes that are typically focused on use of a particular device, together with attendance at live case demonstrations, does not replace the requirement for supervised structured teaching. Stroke is a far more common problem than other neurovascular conditions such as cerebral aneurysm, and the need to train doctors only to do stroke thrombectomy rather than all neurointervention procedures, in order to provide sufficient cover, is currently being reviewed by international societies. It is likely, however, that training of stroke interventionists will need to take place in established neurointervention centres.

\section{Mechanical thrombectomy for stroke in SA}

The new era of endovascular therapy for large-vessel ischaemic stroke is likely to present many challenges in SA. For the trial results to be replicated and patients with acute stroke to reap the benefits of endovascular management, it is important that mechanical thrombectomy starts on a firm foundation. This includes identification and support of comprehensive stroke units with appropriate referral pathways of selected patients from primary stroke centres. One of the distinguishing features of a comprehensive stroke centre is the availability of a qualified neurointerventionist with protocols for thrombectomy. In the private sector, primary stroke units are rapidly being established in response to the advantage offered by new treatments. This is a positive development that should result in better care and improved patient access and includes the effective use of intravenous thrombolytics. However, for the additional benefit of neurointerventional procedures to be realised, we need to ensure that patients have access to comprehensive stroke centres, and that there are sufficient trained stroke interventionists to cover these services. This is certainly possible with the collaboration of all role-players and if established guidelines are followed.

1. Mystroke. http://www.mystroke.co.za (accessed 2 September 2015).

2. Stroke Unit Trialists' Collaboration. Organised inpatient (stroke unit) care for stroke. Cochrane Database Syst Rev 2013, Issue 9. Art. No.: CD000197. DOI:10.1002/14651858.CD000197.pub3

3. Enberson J, Lees KR, Lyden P, et al. Effect of treatment delay, age, and stroke severity on the effects of intravenous thrombolysis with alteplase for acute ischaemic stroke: A meta-analysis of individual patient data from randomized trials. Lancet 2014;384(9958):1929-1935. DOI:10.1016/S0140-6736(14)60584-5

4. Bhatia R, Hill MD, Shobha N, et al. Low rates of acute recanalization with intravenous recombinant tissue plasminogen activator in ischemic stroke. Stroke 2010;41:2254-2258. DOI:10.1161/ STROKEAHA.110.592535

5. Berkhemer OA, Fransen PSS, Beumer D, et al. A randomized trial of intraarterial treatment for acute ischemic stroke. N Engl J Med 2015;372(1):11-20. DOI:10.1056/NEJMoa1411587

6. Campbell BC, Mitchell PJ, Kleinig TJ, et al. Endovascular therapy for ischemic stroke with perfusionimaging selection. N Engl J Med 2015;372(11):1009-1018. DOI:10.1056/NEJMoa1414792

7. Goyal M, Demchuk AM, Menon BK, et al. Randomized assessment of rapid endovascular treatment of 7. Goyal M, Demchuk AM, Menon BK, et al. Randomized assessment of rapid endovascular
ischemic stroke. N Engl J Med 2015;372(11):1019-1030. DOI:10.1056/NEJMoa1414905

8. Saver JL, Goyal M, Bonafe A, et al. Stent-retriever thrombectomy after intravenous t-PA vs t-PA alone in stroke. N Engl J Med 2015;372(24):2285-2295. DOI:10.1056/NEJMoa1415061

9. Jovin TG, Chamorro A, Cobo E, et al. Thrombectomy within 8 hours after symptoms onset in ischemic stroke. N Engl J Med 2015;372(24):2296-306. DOI:10.1056/NEJMoa1503780

10. Grech R, Mizzi A, Pullicino R, et al. Functional outcomes and recanalization rates of stent retrievers in acute ischaemic stroke: A systematic review and meta-analysis. Neuroradiol J 2015;28(2):152-171. DOI:10.1177/1971400915576678

11. Powers WJ, Derdeyn CP, Biller J, et al. 2015 AHA/ASA focused update of the 2013 guidelines for the early management of patients with acute ischemic stroke regarding endovascular treatment: A guideline for healthcare professionals from the American Heart Association/American Stroke Association. Stroke 2015;46(10):3020-3035. DOI:10.1161/STR.0000000000000074

12. Consensus statement on mechanical thrombectomy in acute ischemic stroke - ESO-Karolinska Stroke Update 2014 in collaboration with ESMINT and ESNR. http://2014.strokeupdate.org/consensusstatement-mechanical-thrombectomy-acute-ischemic-stroke (accessed 18 August 2015).

13. Guidelines for training in interventional neuroradiology in South Africa. http://www.sanis.co.za/ 3. Guidelines for training in interventional neuroradiology in South Africa. http://www.sanis.co.za/
Resources/Interventional\%20neuroradiology\%20training\%20doc\%20draft\%203.pdf (accessed 13 November 2015).

Accepted 2 December 2015 